CASE STUDY

\title{
Departmental Action Teams: Empowering Students as Change Agents in Academic Departments
}

\author{
*Daniel L. Reinholza , Alanna Pawlak ${ }^{b}$, Courtney Ngaic, and Mary Pilgrim ${ }^{a}$ \\ ${ }^{\text {a }}$ Department of Mathematics and Statistics, San Diego State University, USA \\ ${ }^{b}$ Center for STEM Learning, University of Colorado Boulder, USA \\ cThe Institute for Learning and Teaching, Colorado State University, USA
}

Contact: daniel.reinholz@sdsu.edu

\section{ABSTRACT}

Supporting and sustaining positive educational change is an area of increasing focus in higher education and remains a persistent challenge. Using student partnerships is one promising way to help promote these much-needed changes. This case study focuses on Departmental Action Teams (DATs), which are groups of faculty, students, and staff working together in the same department to make sustainable improvements to undergraduate education. Here we focus on DATs from four different departments, across two research-intensive universities in the USA, to draw attention to the important roles that students play as change agents in these groups. We also reflect upon the inherent challenges in building partnerships that incorporate meaningful power sharing to effect educational change.

KEYWORDS cultural change, departmental action teams, systemic change, STEM education

Improving undergraduate education remains a pressing challenge. Despite numerous efforts to transform higher education, many efforts are driven by underdeveloped notions of how change works (Beach, Henderson, \& Finkelstein, 2012; Borrego \& Henderson, 2014). Typical approaches consist of developing new teaching techniques or curriculum with little attention to how the techniques will be used by instructors in relation to a larger educational system. Such scale-up approaches have a modest impact at best (e.g., Austin, 2011). Creating meaningful change requires engaging with the university as a system (Kezar, 2011).

Engaging a university holistically requires attention to institutional culture. Culture can be defined as "a historical and evolving set of structures, symbols, people, and resulting power relationships between people" (Reinholz \& Apkarian, 2018, p. 3). Because academic departments have a relatively consistent internal culture, they are seen as a key unit of focus 
for change (Association of American Colleges \& Universities, 2014). Through long-term engagement, departmental cultures can be shifted sustainably, resulting in lasting change (Reinholz et al., 2019).

Students also play a role as change agents in departments (e.g., Dunne \& Zandstra, 2011; Healey, 2016; Hornsby \& Simkins, 2017). Students are key stakeholders for universities, they have their own expertise, and they have resources, connections, and entry points to change that faculty members do not. To contribute to the field of Students as Partners, this article documents successful partnerships between faculty, students, and staff as they work as change agents in the context of Departmental Action Teams (DATs). Because DATs are a research-based model that has been tested on multiple campuses, this line of inquiry has the potential to suggest more generally the role students can play as change agents.

\section{BACKGROUND}

A DAT is a model originally proposed by the authors, and it consists of a group of four to eight faculty, students, and staff that aims to make broad-scale educational improvements within a department (Reinholz, Corbo, Dancy, \& Finkelstein, 2017). A DAT is an externallyfacilitated group that attends to both the outcomes of their work and the process through which that work takes place. The facilitators are typically postdoctoral researchers or members of a Center for Teaching and Learning. DATs create lasting changes by focusing on cross-cutting issues (e.g., curricular alignment, equity, community building) that cannot easily be solved by a single person. With support from leadership, DAT participants work voluntarily on a shared vision for improving undergraduate education in their department.

Students play an important role in DATs. All DATs include at least one student, and many DATs have two or three undergraduate and graduate students as members. Given inherent power dynamics between faculty and students, facilitators aim to promote power sharing among participants (Matthews, 2017). In addition, students are paid a modest stipend, which honors their time and can increase opportunities for students from a variety of backgrounds to participate (Mercer-Mapstone et al., 2017).

DATs are facilitated in accordance with six principles (Quan et al., 2019):

1. Students are partners in the educational process.

2. Work focuses on achieving collective positive outcomes.

3. Data collection, analysis, and interpretation inform decision-making.

4. Collaboration between group members is enjoyable, productive, and rewarding.

5. Continuous improvement is an upheld practice.

6. Work is grounded in a commitment to equity, inclusion, and social justice.

Although students have an influence on how all principles are enacted, principles one and six have an explicit focus on meaningful student partnerships and equity within the group. Principle one draws directly from the Students as Partners literature, and recognizes that students have unique knowledge and expertise to contribute (Hammer \& van Zee, 2006; Robertson, 2015). Principle six focuses on equity, and postulates that diversity among team members adds value to a group and its work (Milliken, Bartel, \& Kurtzberg, 2003). One 
important form of diversity that is often present in DATs is different roles in the department, from student members to tenured faculty. Principle six also promotes equity amongst all voices in a team, to support collective decision-making. To honor these principles and mitigate the power dynamics inherent in heterogeneous groups, DATs develop community standards for group interactions and build a consensus vision to guide their work. In addition, facilitators explicitly focus on process skills, building norms for equitable collaboration and checking in with individual members (e.g., through a coffee meeting) when needed.

\section{METHODS}

This article focuses on DATs at two research-intensive campuses in the USA. To describe how students made these types of contributions to DATs, we used a case study approach, which is appropriate to understand a "contemporary phenomenon in depth and within its reallife context" (Yin, 2009, p. 18). We examined multiple cases to understand how this particular phenomenon manifested in a variety of contexts. Data collection included many forms: detailed meeting minutes, facilitator journal entries, artifacts created by the DATs, interviews and focus groups with DAT participants, and the extensive experience of facilitators (which helped us form impressions of the data).

Here we report on four departments, selected because they provide meaningful examples of student partnerships in DATs. All department names and participant names are pseudonyms; we drew the department pseudonyms (Summoning, Potions, Divination, and Herbs) from the Harry Potter series.

We drew from a theoretical model of students as change agents that emphasizes four roles that students can play (Dunne \& Zandstra, 2011). Drawing upon these four roles, we delineated the types of contributions students make in Table 1, along with examples from DATs.

\section{Table 1: Four roles of student change agents (department pseudonym in parentheses)}

Contribution
Student voice contributes feedback,
perspectives, and experiences unique to
students to form a more complete picture of
the issues at hand.
Decision-making involves students in making
decisions about issues that affect their
community.
Co-creating involves students, faculty, and
staff working together to revise teaching and
learning practices (e.g., curriculum
development).

Actions involve students taking concrete steps to achieve a goal.
Example

Staff and faculty value the contributions of students on the DAT (Summoning).

Students voices contributed meaningfully to DAT decision-making (Potions).

Students co-develop Student Learning Outcomes for a major (Divination).

Students recruit other students to take a draft assessment (Herbs). 


\section{RESULTS}

\section{Potions}

The goal of the Potions DAT was to improve equity within its undergraduate major, because the student population was not very diverse in terms of race and gender. The Potions DAT engaged in a variety of activities, including supporting the department's participation in a campus-wide diversity initiative, contacting admitted students from underrepresented groups, working with university Strategic Relations to develop inclusive advertising materials, connecting with student groups in the department, and creating an equity-focused seminar in the department (Rainey, Corbo, Reinholz, \& Betterton, 2016).

DAT participants described the benefits of having student voices in the DAT. For instance, Cassie, a staff member, described the value of having "a very diverse set of opinions and perspectives." Val, a faculty member, emphasized the value of student voice, stating that, "having both the undergrad and grad student perspectives about both their experiences and what they really care about I think has been really important for shaping what we do, and that's incredibly valuable."

Student members emphasized how the DAT allowed them to contribute to decisionmaking in the department. Willow noted that as a graduate student, the DAT had given her

a way to voice what I'm thinking, because like if I want to change something in the department that's just not going to happen, but if I bring something up here the faculty on this committee have the potential to influence other faculty in the department that I would just never be able to reach and would be terrified to try to talk to anyway.

Willow's statement also draws attention to the inherent power dynamics between students and faculty, as she notes that she would normally have little influence and would be "terrified" to talk with faculty members about change.

Cedric, an undergraduate student, followed up on Willow's statement by noting he felt "empowered" to take actions, and that he wanted to

do these activities, plan, organize, execute. And really, you know, maybe undergrads have a lot more energy, they haven't beaten it out of us yet, but I think we are kind of an untapped potential resource, that it's at least good to have open communication between all these levels. You know, I've been able to effect change already with the LGBT [Lesbian, Gay, Bisexual, Transgender] training that they're going to be starting to do in the department starting fall.

What Cedric describes is being empowered as a part of the DAT, and that would allow him to take actions that can change the department, such as implementing training to raise awareness about the experiences of minoritized populations.

\section{Summoning}

The Summoning DAT focused on building community within its new undergraduate major, through new departmental events and social networking. DAT members perceived that 
the variety of partnerships in the DAT had been crucial to its success. For instance, Bernard, a graduate student, said he "enjoyed" the DAT because of the many "different pools of knowledge" there were to draw from. He described how graduate students are "involved with a lot of different committees," whereas the undergraduates "know a lot about the existing culture within the majors." He continued that the instructors are "really tied to undergraduate education" and "the classroom experience," while faculty know the "official processes" of the department. Together, all of these stakeholders bring useful and complementary skills. This emphasizes the value of student voice.

Kate, a faculty member on the DAT, also emphasized the importance of student voice. She stated that they could not just "assume" what the students want, and then "be upset that they don't show up." She emphasized the need to be proactive in getting students involved, and she saw the DAT as a way to do that. Although the focus of the DAT had been on undergraduate community, Kate felt that the work of the DAT helped them create "a better overall community all the way around" for stakeholder groups in the department.

Despite these positive results, there were still inherent tensions in the group. The undergraduate student member, Sawyer, first described the DAT as "intimidating," but over time he began to understand the DAT as a "community [where] everyone was equal." Sawyer did not elaborate on how his feelings shifted, but we suspect it was supported by explicit efforts of the facilitators, given that in our experience such issues do simply resolve themselves automatically.

Sawyer also described how faculty members on the DAT "suggested" that he present at a faculty meeting, because they thought it would "convince more people" coming from students. This shows how the faculty genuinely embraced student voice, by acknowledging the effect a student voice would have over faculty voice in the context of a faculty meeting and then providing a way for Sawyer's voice to be heard. Sawyer described faculty at the meeting as "very welcoming," and he recognized concrete impacts from this action. For example, he mentioned that another DAT member "created a seminar class specifically as a result of the DAT to help us [students] create resumes." In summary, he stated, "I feel like our voices have been heard, and they're working to their best abilities to try and answer some of those questions, which I think is pretty cool."

\section{Divination}

One goal of the Divination DAT was to revise the Student Learning Outcomes (SLOs) for one of its majors. To date, the DAT has developed SLOs for one of its majors and is designing new assessments for the SLOs. This work was taken up by the whole department during a departmental retreat, and the department decided to create documents to explicitly connect the curriculum to the SLOs.

The facilitator journals for this DAT make frequent mention of interactions between Peyton (a graduate student), Marley (an undergraduate student), and other DAT members. Very early in the DAT's work (just two months after it had formed), the facilitators noted the role of student voice, describing how the faculty members recognized student expertise. "Marley's point of view was considered frequently for determining if the SLO was assessed or not. He's living the experience that they are trying to guess at." A month later, the facilitators 
documented Riley, a faculty member, explicitly valuing the contributions of the students during a meeting, and at the same meeting, the students also recognizing their own value in contributing to the group.

There were also documented instances of Marley "reporting out to the group" from his subgroup meeting and being treated as "an equal member of the DAT." This related to his work in co-creating the SLOs. Marley began to more freely offer his perspectives during meetings, even without being explicitly asked what he thought. It became clear that the students were an important part of the DAT, when nearly a year after the formation of the DAT, one of the faculty members remarked it was "too bad Marley isn't here, we could use his input." Again, we see a group where student voice was explicitly valued and the student members of the DAT became increasingly involved and shared power in the co-creation of the DAT's work.

\section{Herbs}

The Herbs DAT focused on how to assess skills development across the department's major. This DAT has developed and piloted and is implementing a skills assessment as well as creating a department-wide assessment plan. Undergraduate student Jamie described her involvement in co-creation, through "identifying skillsets from syllabi," "coding" the skillsets, and contacting professors to determine the top five skills. Through her work in a summer subgroup of the DAT, Jamie helped develop a draft assessment for the skills and recruited students to take the assessment. On the whole, she noted that the group dynamics were "very comfortable, very friendly," but that she still "felt a little out of place" with PhD students and faculty members. This shows that even in a facilitated group with efforts to meaningfully create an equitable environment, the traditional power structures inherent in student and faculty roles can still persist.

Ellen, a graduate student on the DAT, described how the DAT was "interviewing students about what they want," and the students described having "no software or coding skills," which made it hard for them to compete for jobs. Ellen "brought this to a faculty meeting and now there are initiatives to build this into [their] curriculum." This example shows the importance of student voice, concrete actions such as interviewing students, and involving students in decision-making (through the faculty meeting). We note that students had more decision-making power within the DAT, and this only translated partially to the rest of the department.

Still, Ellen also noted power dynamics, contrasting when she was the only graduate student on the DAT, and when more students were added. She remarked that when more students were added to the DAT, some faculty reverted to traditional modes of interaction, "delegating tasks down to the students more than they delegate to each other, and also being a little bit more resistant when students challenge them." Ellen describes a situation where the presence of more graduate students made it easier for them to be seen as "other" from the faculty, whereas when she was the only student it was easier to be perceived as an equal member of the group. To explain why this happened, Ellen believed it was "just habit," and that "in all other contexts it's totally fine to delegate down to" students. 


\section{DISCUSSION}

This brief description of four focal departments highlights the ways that students can be enabled to act as change agents in a DAT, which provides value to the DAT work. In particular, student voice provided new ways of looking at areas of improvement in undergraduate education, decision-making allowed for students to contribute to structural changes in the departments, co-creation of products resulted in new assessments and learning outcomes, and actions led to concrete activities taking place. The students themselves felt more empowered to engage in change work to improve their departments. All of the DATs we have described made meaningful and lasting improvements to their departments. Moreover, student, faculty, and staff members on the DATs all noted additional benefits from the interactions.

These cases also highlight some of the inherent challenges of involving students as partners in change working groups. Students such as Sawyer and Jamie mentioned how working with faculty could be intimidating, and how it was easy to initially feel out of place. Still, both Sawyer and Jamie described increased feelings of comfort as the DAT progressed, which suggests that facilitation moves in the DATs may help overcome some of the inherent power imbalances. The cases support previous assertions that action should be taken to share power between students and faculty (Matthews, 2017).

We hypothesize that power imbalances may be addressed in multiple ways in the future. Our work suggests that some imbalances arise from a department's existing culture and how it shapes power relations between students and faculty. Thus, we hypothesize that changing other aspects of a department (e.g., student engagement in courses, community building, undergraduate research) could potentially shift the overall culture in a way that supports power sharing in the DAT. We also suspect that faculty members in the study were unaware of how students felt about power imbalances. We hypothesize that sharing this concern in general (e.g., by sharing examples from the students we quoted) and creating opportunities for feedback from students could raise awareness within a DAT and prompt structural and behavioral changes towards equity. As noted in other models of change (e.g., Rogers, 2010) awareness is often the first step towards change, so deliberately including time to reflect on power relations and how they manifest in a DAT could decrease power imbalances. All of these hypotheses need to be tested with future research.

In closing, this article contributes to the Students as Partners literature by drawing attention to the many ways that students can contribute as change agents. Further, we showed how the model from Dunne and Zandstra (2011) can be a useful organizing framework for understanding how students contribute to change efforts. Our work suggests that students can play an important role in supporting systemic change in undergraduate departments, but further research is needed to explore students' participation in DATs across more contexts.

This research was conducted with the approval of an Institutional Review Board at The University of Colorado Boulder. 


\section{ACKNOWLEDGEMENTS}

The authors thank Amelia Stone-Johnstone and Chris Geanious for their feedback on an earlier draft of this manuscript. This material is based on work supported by the National Science Foundation under Grant No 1626565.

\section{NOTE ON CONTRIBUTORS}

Daniel Reinholz is an Assistant Professor of Mathematics Education and Professor of Equity at San Diego State University, where he develops tools to address systemic oppression in STEM education. His research is primarily situated within three interrelated areas: classroom design, racial and gender equity, and systemic change.

Alanna Pawlak is a postdoctoral researcher at the University of Colorado Boulder. Her research focuses on collaborative learning environments in introductory physics, studying areas such as student reasoning, student interactions, and undergraduate learning assistants' approaches to teaching in these environments.

Courtney Ngai a postdoctoral researcher at Colorado State University. Her work includes research into understanding and characterizing how chemistry students classify and differentiate substances. She has also collaborated with science and chemistry teachers affiliated with Boston Public Schools to assess and foster students' chemical thinking.

Mary Pilgrim is an Assistant Professor of Mathematics Education at San Diego State University. Her research focuses on the use of writing to promote metacognition and reflection in mathematics, Graduate Teaching Assistant professional development, and institutional transformation.

No authors were students at the time of this work.

\section{REFERENCES}

Association of American Colleges \& Universities. (2014). Achieving systemic change: A sourcebook for advancing and funding undergraduate STEM education. Washington, DC: Association of American Colleges \& Universities.

Austin, A. E. (2011). Promoting evidence-based change in undergraduate science education. East Lansing, MI: Michigan State University.

Beach, A. L., Henderson, C., \& Finkelstein, N. (2012). Facilitating change in undergraduate STEM education. Change: The Magazine of Higher Learning, 44(6), 52-59. https://doi.org/10.1080/00091383.2012.728955

Borrego, M., \& Henderson, C. (2014). Increasing the use of evidence-based teaching in STEM higher education: A comparison of eight change strategies: Increasing evidence-based 
teaching in STEM education. Journal of Engineering Education, 103(2), 220-252. https://doi.org/10.1002/jee.20040

Dunne, E., \& Zandstra, R. (2011). Students as change agents-New ways of engaging with learning and teaching in higher education. Bristol, UK: A Joint University of Exeter/ESCalate/HE Academy Publication.

Hammer, D., \& van Zee, E. (2006). Seeing the science in children's thinking: Case studies of student inquiry in Physical Science. Portsmouth, NH: Heinemann.

Healey, M. (2016). Students as partners and change agents. Howden, UK: Healey HE Consultants.

Hornsby, K., \& Simkins, S. (2017). Wabash-Provost Scholars Program. Retrieved from https://wpscholars.wordpress.com/

Kezar, A. (2011). What is the best way to achieve broader reach of improved practices in higher education? Innovative Higher Education, 36(4), 235-247. https://doi.org/10.1007/s10755-011-9174-z

Matthews, K. E. (2017). Five propositions for genuine Students as Partners practice. International Journal for Students as Partners, 1(2). https://doi.org/10.15173/ijsap.v1i2.3315

Mercer-Mapstone, L., Dvorakova, S. L., Matthews, K. E., Abbot, S., Cheng, B., Felten, P., Knorr, K., Marquis, E., Shammas, R., \& Swaim, K. (2017). A systematic literature review of students as partners in higher education. International Journal for Students as Partners, 1(1). https://doi.org/10.15173/ijsap.v1i1.3119

Milliken, F. J., Bartel, C. A., \& Kurtzberg, T. R. (2003). Diversity and creativity in work groups: A dynamic perspective on the affective and cognitive processes that link diversity and performance. In P. B. Paulus \& B. A. Nijstad (Eds.), Group Creativity: Innovation Through Collaboration (pp. 32-62). New York, NY: Oxford.

Quan, G. M., Corbo, J. C., Falkenberg, K., Finkelstein, N., Geanious, C., Ngai, C., Pawlak, A., Pilgrim, M. E., Reinholz, D. L., Smith, C., \& Wise, S. (2019). Designing for institutional transformation: Six principles for department-level interventions. Physical Review Physics Education Research, 15(1), 010141. https://doi.org/10.1103/PhysRevPhysEducRes.15.010141

Rainey, K., Corbo, J. C., Reinholz, D. L., \& Betterton, M. (2016). Improving representation in physical sciences using a Departmental Action Team. In D. L. Jones, L. Ding, \& A. Traxler (Eds.), 2016 Physics Education Research Conference Proceedings (pp. 264-267). https://doi.org/10.1119/perc.2016.pr.061

Reinholz, D. L., \& Apkarian, N. (2018). Four frames for systemic change in STEM departments. International Journal of STEM Education, 5(1), 1-10. https://doi.org/10.1186/s40594$\underline{018-0103-x}$

Reinholz, D. L., Corbo, J. C., Dancy, M., \& Finkelstein, N. (2017). Departmental Action Teams: Supporting faculty learning through departmental change. Learning Communities Journal, 9, 5-32.

Reinholz, D. L., Ngai, C., Quan, G., Pilgrim, M. E., Corbo, J. C., \& Finkelstein, N. (2019). Fostering sustainable improvements in science education: An analysis through four frames. Science Education, 103(5), 1125-1150. https://doi.org/10.1002/sce.21526 
Robertson, A. D. (2015). Valuing student ideas morally, instrumentally, and intellectually. In A. Churukian, D. L. Jones, \& L. Ding (Eds.), 2015 Physics Education Research Conference Proceedings, (pp. 275-278). http://dx.doi.org/10.1119/perc.2015.pr.064

Rogers, E. M. (2010). Diffusion of innovations. New York, NY: Simon and Schuster.

Yin, R. K. (2009). Case study research: Design and methods. Thousand Oaks, CA: Sage. 